Article

\title{
Non-Euclidean Geometry, Nontrivial Topology and Quantum Vacuum Effects
}

\author{
Yurii A. Sitenko ${ }^{1, *}$, Volodymyr M. Gorkavenko ${ }^{2}$ \\ 1 Bogolyubov Institute for Theoretical Physics, National Academy of Sciences of Ukraine, \\ 14-b Metrologichna street, 03143 Kyiv, Ukraine \\ 2 Department of Physics, Taras Shevchenko National University of Kyiv, 64 Volodymyrs'ka Street, \\ 01601 Kyiv, Ukraine; gorkavol@gmail.com \\ * Correspondence: yusitenko@bitp.kiev.ua
}

Received: 3 November 2017; Accepted: 25 December 2017; Published: 31 January 2018

\begin{abstract}
Space out of a topological defect of the Abrikosov-Nielsen-Olesen (ANO) vortex type is locally flat but non-Euclidean. If a spinor field is quantized in such a space, then a variety of quantum effects are induced in the vacuum. On the basis of the continuum model for long-wavelength electronic excitations originating in the tight-binding approximation for the nearest-neighbor interaction of atoms in the crystal lattice, we consider quantum ground-state effects in Dirac materials with two-dimensional monolayer structures warped into nanocones by a disclination; the nonzero size of the disclination is taken into account, and a boundary condition at the edge of the disclination is chosen to ensure self-adjointness of the Dirac-Weyl Hamiltonian operator. We show that the quantum ground-state effects are independent of the disclination size, and we find circumstances in which they are independent of parameters of the boundary condition.
\end{abstract}

Keywords: nanocones; ground state; quantum effects in monolayer crystals

\section{Introduction}

Usually, the effects of non-Euclidean geometry are identified with the effects that are due to the curvature of space. It can be immediately shown that this is not the case and that there are spaces that are flat but non-Euclidean; moreover, such spaces are of nontrivial topology.

A simplest example is given by a two-dimensional space (surface) that is obtained from a plane by cutting a segment of a certain angular size and then sewing together the edges. The resulting surface is that which is conical and flat but has a singular point corresponding to the apex of the cone. To be more precise, the intrinsic (Gaussian) curvature of the conical surface is proportional to the two-dimensional delta-function placed at the apex; the coefficient of proportionality is the deficit angle. The topology of the conical surface with a deleted apex is nontrivial: $\pi_{1}=\mathbb{Z}$, where $\pi_{1}$ is the first homotopy group and $\mathbb{Z}$ is the set of integer numbers. Usual cones correspond to positive values of the deficit angle, that is, to the situation when a segment is deleted from the plane. However, one can imagine a situation in which a segment is added to the plane; then the deficit angle is negative, and the resulting flat surface can be denoted as a saddle-like cone. The deleted segment is bounded by the value of $2 \pi$, whereas the added segment is unbounded. Thus, deficit angles for possible conical surfaces range from $-\infty$ to $2 \pi$.

It is evident that an apex of the conical surface with a positive deficit angle can play the role of a convex lens, whereas an apex of the conical surface with a negative deficit angle can play the role of a concave lens. In fact, two parallel trajectories coming from infinity towards the apex from different sides of it, after bypassing it, converge (and intersect) in the case of the positive deficit angle and diverge in the case of the negative deficit angle. This demonstrates the non-Euclidean nature of conical surfaces, providing a basis for understanding such physical objects as cosmic strings. 
Conical space emerges inevitably as an outer space of any topological defect in the form of the Abrikosov-Nielsen-Olesen (ANO) vortex [1,2]. Topological defects are produced as a consequence of phase transitions with the spontaneous breakdown of continuous symmetry in various physical systems, in particular, in superfluids, superconductors and liquid crystals. Cosmic strings with a specific gravitational lensing effect (doubling the image of an astrophysical object) are the ANO vortices that are produced as a result of phase transitions at the early stage of the evolution of the universe; see reviews in [3,4]. Otherwise, in micro- and nano-physics, a wealth of new phenomena, suggesting possible applications to technology and industry, are promised by a synthesis in this century of strictly two-dimensional atomic crystals (e.g., a monolayer of carbon atoms-graphene [5,6]). Topological defects (disclinations) on such layers are similar to the transverse sections of cosmic strings. A disclination warps a sheet of a layer, rolling it into a nanocone; moreover, a physically meaningful range of values of the deficit angle is extended to also include negative values that correspond to saddle-like cones or cosmic strings with negative tension.

While considering the effect of the ANO vortex on the vacuum of quantum matter, the following circumstance should be taken into account: because the vacuum of quantum matter exists outside the ANO vortex core, an issue of the choice of boundary conditions at the edge of the core is of primary importance. The most general boundary condition for the matter wave function at the core edge is given by requiring self-adjointness of the Hamiltonian operator (energy operator in first-quantized theory).

In the present paper, we consider quantum effects that are induced in the vacuum of the second-quantized pseudorelativistic gapless (i.e., massless) spinor field in $(2+1)$-dimensional space-time which is a section orthogonal to the ANO vortex axis; hence the Hamiltonian operator takes the form $H=-i \boldsymbol{\alpha} \cdot \boldsymbol{\nabla}$, where the covariant derivative $\boldsymbol{\nabla}$ includes both the affine and bundle connections (natural units $\hbar=v_{F}=1$ are used, with the Fermi velocity, $v_{F}$, becoming the velocity of light, $c$, in the truly relativistic case). Condensed matter systems with such a behavior of low-energy electronic excitations are known as the two-dimensional Dirac materials comprising a diverse set ranging from honeycomb crystalline structures (graphene [5], silicene and germanene [7]) to high-temperature $d$-wave superconductors, superfluid phases of helium-3 and topological insulators; see review in [8]. We focus on the quantum ground-state effects (induced $R$-current and pseudomagnetic field) of electronic excitations in graphitic nanocones, although our consideration is quite general to also be relevant for nanocones of the nongraphitic nature; the finite size of a disclination at the conical apex is taken into account.

\section{Continuum Model Description of Electronic Excitations in Monolayer Atomic Crystals with a Disclination}

The squared length element of the conical surface is

$$
d s^{2}=d r^{2}+v^{-2} r^{2} d \varphi^{2}
$$

where $v=(1-\eta)^{-1}$ and $2 \pi \eta$ is the deficit angle. In the case of cosmic strings, the present-day astrophysical observations restrict the values of parameter $\eta$ to the range $0<\eta<10^{-6}$ (see, e.g., [9]). A natural way of producing local curvature in the honeycomb lattice of graphene, silicene or germanene is by substituting some of the hexagons by pentagons (positive curvature) and heptagons (negative curvature). Thus, in the case of crystalline nanocones, the parameter $\eta$ takes discrete values: $\eta=N_{d} / 6$, where $N_{d}$ is an integer that is smaller than 6 . A disclination in the honeycomb lattice results from a substitution of a hexagon by a polygon with $6-N_{d}$ sides; polygons with $N_{d}>0\left(N_{d}<0\right)$ induce locally positive (negative) curvature, whereas the crystalline sheet is flat away from the disclination as is the conical surface away from the apex. In the case of nanocones with $N_{d}>0$, the value of $N_{d}$ is related to the apex angle $\delta, \sin \frac{\delta}{2}=1-\frac{N_{d}}{6}$, and $N_{d}$ counts the number of sectors of the value of $\pi / 3$ that are removed from the crystalline sheet. If $N_{d}<0$, then $-N_{d}$ counts the number of such sectors that are inserted into the crystalline sheet. Certainly, polygonal defects with $N_{d}>1$ and $N_{d}<-1$ are mathematical abstractions as are cones with a point-like apex. In reality, the defects are 
smoothed, and $N_{d}>0$ counts the number of pentagonal defects that are tightly clustered, producing a conical shape; graphitic nanocones with the apex angles $\delta=112.9^{\circ}, 83.6^{\circ}, 60.0^{\circ}, 38.9^{\circ}, 19.2^{\circ}$, which correspond to the values $N_{d}=1,2,3,4,5$, were observed experimentally; see [10] and references therein. Theory also predicts an infinite series of the saddle-like nanocones with quantity $-N_{d}$ counting the number of heptagonal defects that are clustered in their central regions. Saddle-like nanocones serve as an element that is necessary for joining parts of carbon nanotubes of differing radii. On the basis of the long-wavelength continuum model originating in the tight-binding approximation for the nearest-neighbor interactions in the honeycomb crystalline lattice, it was proved [11] that the bundle connection effectively appears in addition to the affine connection of the nanocone, and the Hamiltonian operator takes the form

$$
H=-\mathrm{i}\left[\alpha^{r}\left(\partial_{r}+\frac{1}{2 r}\right)+\alpha^{\varphi}\left(\partial_{\varphi}-\mathrm{i} \frac{\Phi}{2 \pi}\right)\right]
$$

where

$$
\Phi=3 \pi\left(1-v^{-1}\right) R
$$

and matrix $R$ exchanges the sublattice indices, as well as the valley indices, and commutes with $H$ (Equation (2)). We note that in the case of a cosmic string quantity $\Phi$ is the flux of a gauge vector field corresponding to the generator of a spontaneously broken continuous symmetry. Both $R$ and $\alpha$-matrices can be chosen in the block-diagonal form:

$$
R=\left(\begin{array}{cc}
I & 0 \\
0 & -I
\end{array}\right), \quad \alpha^{r}=\alpha_{r}=-\left(\begin{array}{cc}
\sigma^{2} & 0 \\
0 & \sigma^{2}
\end{array}\right), \quad \alpha^{\varphi}=\frac{v}{r}\left(\begin{array}{cc}
\sigma^{1} & 0 \\
0 & -\sigma^{1}
\end{array}\right), \quad \alpha_{\varphi}=\frac{r^{2}}{v^{2}} \alpha^{\varphi}
$$

( $\sigma^{j}$ with $j=1,2,3$ are the Pauli matrices). The solution to the stationary Dirac-Weyl equation, $H \psi_{E}(\mathbf{x})=E \psi_{E}(\mathbf{x})$, is decomposed as

$$
\psi_{E}(\mathbf{x})=\sum_{n \in \mathbb{Z}}\left(\begin{array}{c}
f_{n}^{+}(r, E) \mathrm{e}^{\mathrm{i}(n+1 / 2) \varphi} \\
g_{n}^{+}(r, E) \mathrm{i}^{\mathrm{i}(n+1 / 2) \varphi} \\
f_{n}^{-}(r, E) \mathrm{e}^{\mathrm{i}(n-1 / 2) \varphi} \\
g_{n}^{-}(r, E) \mathrm{i}^{\mathrm{i}(n-1 / 2) \varphi}
\end{array}\right)
$$

where the radial function satisfies the system of first-order differential equations:

$$
\left\{\begin{array}{c}
{\left[-\partial_{r}+\frac{1}{r}( \pm v n-v+1)\right] f_{n}^{ \pm}(r, E)=E g_{n}^{ \pm}(r, E)} \\
{\left[\partial_{r}+\frac{1}{r}( \pm v n-v+2)\right] g_{n}^{ \pm}(r, E)=E f_{n}^{ \pm}(r, E)}
\end{array}\right\}
$$

We consider nanocones with $N_{d}=1,2,3,4,5 \quad(1<v<7)$, as well as with $N_{d}=-1,-2,-3$ $\left(\frac{3}{5}<v<1\right)$, and introduce the following positive quantity:

$$
F=\frac{3}{2} v-\frac{1}{2} v \operatorname{sgn}(v-1)-1
$$

which exceeds 1 at $N_{d}=3,4,5 \quad(2 \leq v<7)$ only; here, $\operatorname{sgn}(u)$ is the sign function. From the whole variety of quantum effects in the ground state of electronic excitations (see [11-13]), our focus is on the induced specific current ( $R$-current), which is defined by the following expression:

$$
\mathbf{j}(\mathbf{x})=-\frac{1}{2} \int_{-\infty}^{\infty} d E|E| \psi_{E}^{\dagger}(\mathbf{x}) \boldsymbol{\alpha} R \psi_{E}(\mathbf{x})
$$


The pseudomagnetic field strength, $\mathbf{B}_{\mathrm{I}}(\mathbf{x})$, is also induced in the ground state, as a consequence of the analogue of the Maxwell equation:

$$
\partial \times \mathbf{B}_{\mathrm{I}}(\mathbf{x})=e \mathbf{j}(\mathbf{x})
$$

Using Equations (4) and (5), one obtains $j_{r}=0$, and the only component of the induced ground-state current:

$$
j_{\varphi}(r)=-\frac{r}{v} \int_{-\infty}^{\infty} d E|E| \sum_{n \in \mathbb{Z}}\left[f_{n}^{+}(r, E) g_{n}^{+}(r, E)+f_{n}^{-}(r, E) g_{n}^{-}(r, E)\right]
$$

is independent of the angular variable. The induced ground-state field strength is also independent of the angular variable, being directed orthogonally to the conical surface:

$$
B_{\mathrm{I}}(r)=e v \int_{r}^{r_{\max }} \frac{d r^{\prime}}{r^{\prime}} j_{\varphi}\left(r^{\prime}\right)+B_{\mathrm{I}}\left(r_{\max }\right)
$$

with the total flux

$$
\Phi_{\mathrm{I}}=\frac{2 \pi}{v} \int_{r_{0}}^{r_{\max }} d r r B_{\mathrm{I}}(r)
$$

where it is assumed without loss of generality that a nanocone is of a rotationally invariant shape with $r_{\max }$ being its radius and $r_{0}$ being the radius of a disclination, $r_{\max } \gg r_{0}$.

\section{Self-Adjointness and Choice of Boundary Conditions}

We note first that Equation (2) is not enough to define the Hamiltonian operator rigorously in a mathematical sense. To define an operator in an unambiguous way, one has to specify its domain of definition. We let the set of functions $\psi$ be the domain of the definition of the operator $H$ and the set of functions $\tilde{\psi}$ be the domain of definition of its adjoint operator $\mathrm{H}^{+}$. Then the operator is Hermitian (or symmetric in mathematical parlance):

$$
\int_{X} d^{2} x \sqrt{g} \tilde{\psi}^{\dagger}(H \psi)=\int_{X} d^{2} x \sqrt{g}\left(H^{\dagger} \tilde{\psi}\right)^{\dagger} \psi
$$

if relation

$$
-\mathrm{i} \int_{\partial X} d l \tilde{\psi}^{\dagger} \alpha \psi=0
$$

is valid; here the functions $\psi(\mathbf{x})$ and $\tilde{\psi}(\mathbf{x})$ are defined in space $X$ with boundary $\partial X$. It is evident that Equation (14) can be satisfied by imposing different boundary conditions for $\psi$ and $\tilde{\psi}$. However, a nontrivial task is to find a possibility that a boundary condition for $\tilde{\psi}$ is the same as that for $\psi$; then the domain of definition of $\mathrm{H}^{+}$coincides with that of $H$, and operator $H$ is self-adjoint (for a review of the Weyl-von Neumann theory of self-adjoint operators, see [14,15]). The action of a self-adjoint operator results in functions belonging to its domain of definition only, and a multiple action and functions of such an operator, for instance, the resolvent and evolution operators, can be consistently defined. Thus, in the case of a surface of radius $r_{\max }$ with a deleted central disc of radius $r_{0}$, we have to ensure the validity of the relations

$$
\left.\tilde{\psi}^{\dagger} \alpha^{r} \psi\right|_{r=r_{0}}=0,\left.\quad \tilde{\psi}^{\dagger} \alpha^{r} \psi\right|_{r=r_{\max }}=0
$$

meaning that the quantum matter excitations do not penetrate outside. It is implied that the functions $\psi$ and $\tilde{\psi}$ are differentiable and square-integrable. As $r_{\max } \rightarrow \infty$, they conventionally turn into 
differentiable functions corresponding to the continuum, and the condition at $r=r_{\text {max }}$ yields no restriction at $r_{\max } \rightarrow \infty$, whereas the condition at $r=r_{0}$ yields

$$
\left.\psi\right|_{r=r_{0}}=\left.\left.K \psi\right|_{r=r_{0}} \quad \tilde{\psi}\right|_{r=r_{0}}=\left.K \tilde{\psi}\right|_{r=r_{0}},
$$

where $K$ is a matrix (element of the Clifford algebra with generators $\alpha^{r}, \alpha^{\varphi}$ and $\beta$ ), which obeys the condition

$$
K^{2}=I
$$

and without loss of generality can be chosen to be Hermitian; in addition, it has to obey either the condition

$$
\left[K, \alpha^{r}\right]_{+}=0
$$

or the condition

$$
\left[K, \alpha^{r}\right]_{-}=0
$$

One can simply go through four linearly independent elements of the Clifford algebra and find that two of them satisfy Equation (18) and the other two satisfy Equation (19). However, if one chooses

$$
K=c_{1} I+c_{2} \alpha^{r}
$$

to satisfy Equation (19), then Equation (17) is violated. The only possibility remains to choose

$$
K=c_{1} \beta+c_{2} \mathrm{i} \beta \alpha^{r}
$$

with real coefficients obeying the condition

$$
c_{1}^{2}+c_{2}^{2}=1
$$

Then both Equations (17) and (18) are satisfied. Using a clear parametrization:

$$
c_{1}=\sin \theta, \quad c_{2}=\cos \theta
$$

we finally obtain

$$
K=\mathrm{i} \beta \alpha^{r} e^{-\mathrm{i} \theta \alpha^{r}}
$$

Thus, the boundary condition (Equation (16)) with $K$ given by Equation (23) is the most general boundary condition ensuring self-adjointness of the Hamiltonian operator on a surface with a deleted disc of radius $r_{0}$, and parameter $\theta$ can be interpreted as the self-adjoint extension parameter. The value $\theta=0$ corresponds to the MIT bag boundary condition, which was proposed as the condition ensuring the confinement of the matter field, that is, the absence of the matter flux across the boundary [16]. However, it should be noted that a condition with an arbitrary value of $\theta$ is motivated equally as well as that with $\theta=0$.

Imposing the boundary condition of Equation (16) with matrix $K$ (Equation (23)) on the solution to the Dirac-Weyl equation, $\psi_{E}(\mathbf{x})$ (Equation (5)), we obtain the condition for the modes:

$$
\cos \left(\frac{\theta}{2}+\frac{\pi}{4}\right) f_{n}^{ \pm}\left(r_{0}, E\right)=-\sin \left(\frac{\theta}{2}+\frac{\pi}{4}\right) g_{n}^{ \pm}\left(r_{0}, E\right)
$$

We compare this with the case of an infinitely thin (point-like) disclination, which was considered in detail in [11-13]. In the latter case, several partial Hamiltonian operators are self-adjoint extended, and the deficiency index can be $(0,0)$ (no need for extension; all partial operators are essentially self-adjoint), $(1,1)$ (one partial operator is extended with one parameter), $(2,2)$ (two partial operators are extended with four parameters), and so forth. In particular, in the case of carbon nanocones, there 
is no need for self-adjoint extension for $N_{d}=3,4,5$, there is one self-adjoint extension parameter for $N=2,1,-1,-2,-3,-6$, there are four or more self-adjoint extension parameters for $N_{d}=-4,-5$ and $N_{d} \leq-7$. For the deficiency index equal to $(1,1)$, the boundary condition at the location of a point-like disclination $(r=0)$ takes the form

$$
\lim _{r \rightarrow 0}\left(\frac{r}{r_{\max }}\right)^{F} \cos \left(\frac{\Theta}{2}+\frac{\pi}{4}\right) f_{n_{\mathrm{c}}}^{ \pm}(r, E)=-\lim _{r \rightarrow 0}\left(\frac{r}{r_{\max }}\right)^{1-F} \sin \left(\frac{\Theta}{2}+\frac{\pi}{4}\right) g_{n_{\mathrm{c}}}^{ \pm}(r, E)
$$

where $\Theta$ is the self-adjoint extension parameter, $F$ is given by Equation (7) for $N_{d}=2,1,-1,-2,-3$ and $F=1 / 2$ for $N_{d}=-6$, while $n_{c}= \pm \frac{1}{2}[\operatorname{sgn}(v-1)-1]$ for $N_{d}=2,1,-1,-2,-3$ and $n_{c}=\mp 2$ for $N_{d}=-6$. As follows from the present section, in the case of a disclination of nonzero size, when the boundary condition is imposed at its edge, the total Hamiltonian operator is self-adjoint extended with the use of one parameter; see Equation (24).

\section{Quantum Effects in the Ground State of Electronic Excitations in Nanocones}

Using the explicit form of modes $f_{n}^{ \pm}$and $g_{n}^{ \pm}$, satisfying Equations (6) and (24), we calculate the current (Equation (10)) and field strength (Equation (11)). In the case of $\frac{3}{5}<v<2(0<F<1)$, we obtain

$$
\begin{aligned}
& \left.j_{\varphi}(r)\right|_{F<\frac{1}{2}, \theta \neq-\frac{\pi}{2}}=-\frac{1}{(2 \pi)^{2}} \frac{1}{r}\left\{\int_{0}^{\infty} \frac{d u}{\cosh ^{2}(u / 2)}\right. \\
& \times \frac{\left.\sin (F \pi) \cosh \left[\left(F+v-\frac{1}{2}\right) u\right]-\sin [(F+v) \pi)\right] \cosh \left[\left(F-\frac{1}{2}\right) u\right]}{\cosh (v u)-\cos (v \pi)} \\
& +8 r^{2} \int_{0}^{\infty} d q q\left[\sum_{l=0}^{\infty} C_{v l+1-F}^{(\wedge)}\left(q r_{0}\right) K_{v l+1-F}(q r) K_{v l-F}(q r)\right. \\
& \left.\left.-\sum_{l=1}^{\infty} C_{v l+F}^{(\vee)}\left(q r_{0}\right) K_{v l+F}(q r) K_{v l-1+F}(q r)\right]\right\} \\
& \left.j_{\varphi}(r)\right|_{F>\frac{1}{2}, \theta \neq \frac{\pi}{2}}=\frac{1}{(2 \pi)^{2}} \frac{1}{r}\left\{\int_{0}^{\infty} \frac{d u}{\cosh ^{2}(u / 2)}\right. \\
& \times \frac{\left.\sin (F \pi) \cosh \left[\left(F-v-\frac{1}{2}\right) u\right]-\sin [(F-v) \pi)\right] \cosh \left[\left(F-\frac{1}{2}\right) u\right]}{\cosh (v u)-\cos (v \pi)} \\
& -8 r^{2} \int_{0}^{\infty} d q q\left[\sum_{l=1}^{\infty} C_{v l+1-F}^{(\wedge)}\left(q r_{0}\right) K_{v l+1-F}(q r) K_{v l-F}(q r)\right. \\
& \left.\left.-\sum_{l=0}^{\infty} C_{v l+F}^{(\vee)}\left(q r_{0}\right) K_{v l+F}(q r) K_{v l-1+F}(q r)\right]\right\}
\end{aligned}
$$




$$
\begin{aligned}
\left.j_{\varphi}(r)\right|_{F \neq \frac{1}{2}, \theta= \pm \frac{\pi}{2}}=\mp \frac{1}{2(2 \pi)^{2}} \frac{1}{r}\left\{\int_{0}^{\infty} \frac{d u}{\cosh ^{2}(u / 2)}\right. \\
\times \frac{\sin (F \pi) \cosh \left[\left(F-\frac{1}{2} \pm v\right) u\right]-\sin [(F \pm v) \pi] \cosh \left[\left(F-\frac{1}{2}\right) u\right]}{\cosh (v u)-\cos (v \pi)} \\
+8 r^{2} \int_{0}^{\infty} d q q\left[\frac{I_{\frac{1}{2} \mp\left(F-\frac{1}{2}\right)}\left(q r_{0}\right)}{K_{\frac{1}{2} \mp\left(F-\frac{1}{2}\right)}\left(q r_{0}\right)} K_{F}(q r) K_{1-F}(q r)\right. \\
+\sum_{l=1}^{\infty}\left(\frac{I_{v l-F+\frac{1}{2} \pm \frac{1}{2}\left(q r_{0}\right)}}{K_{v l-F+\frac{1}{2} \pm \frac{1}{2}}\left(q r_{0}\right)} K_{v l+1-F}(q r) K_{v l-F}(q r)\right. \\
\left.j_{\varphi}(r)\right|_{F=\frac{1}{2}}=-\frac{\sin \theta}{2 \pi^{2}}\left[\frac{1}{r-r_{0}}+8 r \int_{0}^{\infty} d q q \sum_{l=1}^{\infty} \tilde{C}_{v l+\frac{1}{2}}\left(q r_{0}\right) K_{v l+\frac{1}{2}}(q r) K_{v l-\frac{1}{2}}(q r)\right]
\end{aligned}
$$

$$
\begin{aligned}
\left.B_{\mathrm{I}}(r)\right|_{F<\frac{1}{2}, \theta \neq-\frac{\pi}{2}}= & -\frac{v e}{(2 \pi)^{2}} \frac{1}{r}\left\{\int_{0}^{\infty} \frac{d u}{\cosh ^{2}(u / 2)}\right. \\
\times & \frac{\left.\sin (F \pi) \cosh \left[\left(F+v-\frac{1}{2}\right) u\right]-\sin [(F+v) \pi)\right] \cosh \left[\left(F-\frac{1}{2}\right) u\right]}{\cosh (v u)-\cos (v \pi)} \\
& +8 r \int_{r}^{r_{\max }} d r^{\prime} \int_{0}^{\infty} d q q\left[\sum_{l=0}^{\infty} C_{v l+1-F}^{(\wedge)}\left(q r_{0}\right) K_{v l+1-F}\left(q r^{\prime}\right) K_{v l-F}\left(q r^{\prime}\right)\right. \\
- & \left.\left.-\sum_{l=1}^{\infty} C_{v l+F}^{(v)}\left(q r_{0}\right) K_{v l+F}\left(q r^{\prime}\right) K_{v l-1+F}\left(q r^{\prime}\right)\right]\right\}
\end{aligned}
$$

$$
\begin{aligned}
\left.B_{\mathrm{I}}(r)\right|_{F>\frac{1}{2}, \theta \neq \frac{\pi}{2}}= & \frac{v e}{(2 \pi)^{2}} \frac{1}{r}\left\{\int_{0}^{\infty} \frac{d u}{\cosh ^{2}(u / 2)}\right. \\
\times & \frac{\left.\sin (F \pi) \cosh \left[\left(F-v-\frac{1}{2}\right) u\right]-\sin [(F-v) \pi)\right] \cosh \left[\left(F-\frac{1}{2}\right) u\right]}{\cosh (v u)-\cos (v \pi)} \\
& -8 r \int_{r}^{r_{\max }} d r^{\prime} \int_{0}^{\infty} d q q\left[\sum_{l=1}^{\infty} C_{v l+1-F}^{(\wedge)}\left(q r_{0}\right) K_{v l+1-F}\left(q r^{\prime}\right) K_{v l-F}\left(q r^{\prime}\right)\right. \\
& \left.\left.-\sum_{l=0}^{\infty} C_{v l+F}^{(\vee)}\left(q r_{0}\right) K_{v l+F}\left(q r^{\prime}\right) K_{v l-1+F}\left(q r^{\prime}\right)\right]\right\}
\end{aligned}
$$




$$
\begin{array}{r}
\left.B_{\mathrm{I}}(r)\right|_{F \neq \frac{1}{2}, \theta= \pm \frac{\pi}{2}}=\mp \frac{v e}{(2 \pi)^{2}} \frac{1}{r}\left\{\int_{0}^{\infty} \frac{d u}{\cosh ^{2}(u / 2)}\right. \\
\times \frac{\sin (F \pi) \cosh \left[\left(F-\frac{1}{2} \pm v\right) u\right]-\sin [(F \pm v) \pi] \cosh \left[\left(F-\frac{1}{2}\right) u\right]}{\cosh (v u)-\cos (v \pi)} \\
+8 r \int_{r}^{r_{\max } d r^{\prime} \int_{0}^{\infty} d q q\left[\frac{I_{\frac{1}{2} \mp\left(F-\frac{1}{2}\right)}\left(q r_{0}\right)}{K_{\frac{1}{2} \mp\left(F-\frac{1}{2}\right)}\left(q r_{0}\right)} K_{F}\left(q r^{\prime}\right) K_{1-F}\left(q r^{\prime}\right)\right.} \\
+\sum_{l=1}^{\infty}\left(\frac{I_{v l-F+\frac{1}{2} \pm \frac{1}{2}}\left(q r_{0}\right)}{K_{v l-F+\frac{1}{2} \pm \frac{1}{2}}\left(q r_{0}\right)} K_{v l+1-F}\left(q r^{\prime}\right) K_{v l-F}\left(q r^{\prime}\right)\right. \\
\left.\left.\left.\quad+\frac{I_{v l+F-\frac{1}{2} \mp \frac{1}{2}}\left(q r_{0}\right)}{K_{v l+F-\frac{1}{2} \mp \frac{1}{2}}\left(q r_{0}\right)} K_{v l+F}\left(q r^{\prime}\right) K_{v l-1+F}\left(q r^{\prime}\right)\right)\right]\right\}
\end{array}
$$

and

$$
\left.B_{\mathrm{I}}(r)\right|_{F=\frac{1}{2}}=\frac{v e \sin \theta}{2 \pi^{2}}\left[\frac{1}{r_{0}} \ln \left(1-\frac{r_{0}}{r}\right)-8 \int_{r}^{r_{\max }} d r^{\prime} \int_{0}^{\infty} d q q \sum_{l=1}^{\infty} \tilde{C}_{v l+\frac{1}{2}}\left(q r_{0}\right) K_{v l+\frac{1}{2}}\left(q r^{\prime}\right) K_{v l-\frac{1}{2}}\left(q r^{\prime}\right)\right]
$$

where

$$
\begin{aligned}
C_{\rho}^{(\wedge)}(v)=\left\{I_{\rho}(v) K_{\rho}(v) \tan \left(\frac{\theta}{2}+\frac{\pi}{4}\right)-\right. & \left.I_{\rho-1}(v) K_{\rho-1}(v) \cot \left(\frac{\theta}{2}+\frac{\pi}{4}\right)\right\} \\
\times & {\left[K_{\rho}^{2}(v) \tan \left(\frac{\theta}{2}+\frac{\pi}{4}\right)+K_{\rho-1}^{2}(v) \cot \left(\frac{\theta}{2}+\frac{\pi}{4}\right)\right]^{-1} } \\
C_{\rho}^{(\vee)}(v)=\left\{I_{\rho}(v) K_{\rho}(v) \cot \left(\frac{\theta}{2}+\frac{\pi}{4}\right)-\right. & \left.I_{\rho-1}(v) K_{\rho-1}(v) \tan \left(\frac{\theta}{2}+\frac{\pi}{4}\right)\right\} \\
\times & {\left[K_{\rho}^{2}(v) \cot \left(\frac{\theta}{2}+\frac{\pi}{4}\right)+K_{\rho-1}^{2}(v) \tan \left(\frac{\theta}{2}+\frac{\pi}{4}\right)\right]^{-1} }
\end{aligned}
$$

and

$$
\tilde{C}_{v l+\frac{1}{2}}(v)=\frac{2}{v} \frac{K_{v l+\frac{1}{2}}(v) K_{v l-\frac{1}{2}}(v)}{\cos ^{2} \theta\left[K_{v l+\frac{1}{2}}^{2}(v)+K_{v l-\frac{1}{2}}^{2}(v)\right]^{2}+4 \sin ^{2} \theta K_{v l+\frac{1}{2}}^{2}(v) K_{v l-\frac{1}{2}}^{2}(v)}
$$

$I_{\rho}(u)$ and $K_{\rho}(u)$ are the modified Bessel functions with the exponential increase and decrease, respectively, at large real positive values of their argument.

In the case of $2 \leq v<7 \quad(F=v-1)$, we obtain

$$
\begin{aligned}
j_{\varphi}(r)=-\frac{1}{(2 \pi)^{2}} \frac{1}{r}\left\{\frac{\pi}{v} \sum_{p=1}^{\nabla v / 2 \rrbracket} \frac{\sin (3 p \pi / v)}{\sin ^{2}(p \pi / v)}+\sin (v \pi) \int_{0}^{\infty} \frac{d u}{\cosh ^{2}(u / 2)} \frac{\cosh \left(\frac{3}{2} u\right)}{\cosh (v u)-\cos (v \pi)}\right. \\
+8 r^{2} \int_{0}^{\infty} d q q\left[\sum_{l=1}^{\infty} C_{v(l-1)+2}^{(\wedge)}\left(q r_{0}\right) K_{v(l-1)+2}(q r) K_{v(l-1)+1}(q r)\right. \\
\left.\left.\quad-\sum_{l=0}^{\infty} C_{v(l+1)-1}^{(\vee)}\left(q r_{0}\right) K_{v(l+1)-1}(q r) K_{v(l+1)-2}(q r)\right]\right\}
\end{aligned}
$$

and 


$$
\begin{aligned}
B_{\mathrm{I}}(r)=-\frac{v e}{(2 \pi)^{2}} \frac{1}{r}\left\{\frac{\pi}{v} \sum_{p=1}^{\square v / 2 \rrbracket} \frac{\sin (3 p \pi / v)}{\sin ^{2}(p \pi / v)}+\sin (v \pi) \int_{0}^{\infty} \frac{d u}{\cosh ^{2}(u / 2)} \frac{\cosh \left(\frac{3}{2} u\right)}{\cosh (v u)-\cos (v \pi)}\right. \\
+8 r \int_{r}^{r_{\max }} d r^{\prime} \int_{0}^{\infty} d q q\left[\sum_{l=1}^{\infty} C_{v(l-1)+2}^{(\wedge)}\left(q r_{0}\right) K_{v(l-1)+2}\left(q r^{\prime}\right) K_{v(l-1)+1}\left(q r^{\prime}\right)\right. \\
\left.\left.-\sum_{l=0}^{\infty} C_{v(l+1)-1}^{(\vee)}\left(q r_{0}\right) K_{v(l+1)-1}\left(q r^{\prime}\right) K_{v(l+1)-2}\left(q r^{\prime}\right)\right]\right\}
\end{aligned}
$$

where $\llbracket u \rrbracket$ is the integer part of quantity $u$ (i.e., the integer that is less than or equal to $u$ ), and $p$ denotes a positive integer.

It should be noted that the integral over the $q$ variable in Equations (26)-(29) and (37) vanishes in the limit of $r_{0} \rightarrow 0$. Moreover, in the limit of $r \rightarrow \infty$, it decreases at least as $r^{-2 \rho}$, where

$$
\begin{aligned}
& \rho=2-F, \quad \overline{5}<v<2, \quad\left\{\begin{array}{l}
0<F<\frac{1}{2}, \quad \theta \neq-\frac{\pi}{2} \\
\frac{1}{2}<F<1, \quad \theta=\frac{\pi}{2}
\end{array}\right\} \\
& \rho=1+F, \quad \frac{3}{5}<v<2, \quad\left\{\begin{array}{ll}
\frac{1}{2}<F<1, & \theta \neq \frac{\pi}{2} \\
0<F<\frac{1}{2}, & \theta=-\frac{\pi}{2}
\end{array}\right\} \\
& \rho=v+\frac{1}{2}, \quad \frac{3}{5}<v<2, \quad F=\frac{1}{2} \\
& \left\{\begin{array}{l}
\rho=1+\frac{\ln \ln r}{2 \ln r}, \quad v=2 \\
\rho=v-1, \quad 2<v<7
\end{array}\right\}, \quad F=v-1
\end{aligned}
$$

The latter circumstance has far-reaching consequences, when we turn to the total flux of the induced ground-state field strength; see Equation (12). Namely, the contribution of the $q$-integral to $\Phi_{\mathrm{I}}$ is damped and the field strength is proportional to the current in the physically sensible case, that is, at $r_{\max } \gg r_{0}$ :

$$
j_{\varphi}(r)=\frac{\Phi_{\mathrm{I}}}{2 \pi e r_{\max }} \frac{1}{r}, \quad B_{\mathrm{I}}(r)=\frac{v \Phi_{\mathrm{I}}}{2 \pi r_{\max }} \frac{1}{r}
$$

where

$$
\begin{aligned}
& \left.\Phi_{\mathrm{I}}\right|_{0<F<\frac{1}{2}, \theta \neq-\frac{\pi}{2}}=\left.\Phi_{\mathrm{I}}\right|_{\frac{1}{2}<F<1, \theta=\frac{\pi}{2}}=-\frac{e}{2 \pi} r_{\max } \int_{0}^{\infty} \frac{d u}{\cosh ^{2}(u / 2)} \\
& \times \frac{\left.\sin (F \pi) \cosh \left[\left(F+v-\frac{1}{2}\right) u\right]-\sin [(F+v) \pi)\right] \cosh \left[\left(F-\frac{1}{2}\right) u\right]}{\cosh (v u)-\cos (v \pi)}+O\left(r_{\max }^{-1+2 F}\right), \quad \frac{3}{5}<v<2 \\
& \left.\Phi_{\mathrm{I}}\right|_{\frac{1}{2}<F<1, \theta \neq \frac{\pi}{2}}=\left.\Phi_{\mathrm{I}}\right|_{0<F<\frac{1}{2}, \theta=-\frac{\pi}{2}}=\frac{e}{2 \pi} r_{\max } \int_{0}^{\infty} \frac{d u}{\cosh ^{2}(u / 2)} \\
& \times \frac{\left.\sin (F \pi) \cosh \left[\left(F-v-\frac{1}{2}\right) u\right]-\sin [(F-v) \pi)\right] \cosh ^{2}\left[\left(F-\frac{1}{2}\right) u\right]}{\cosh (v u)-\cos (v \pi)}+O\left(r_{\max }^{-2 F+1}\right), \quad \frac{3}{5}<v<2 \\
& \left.\Phi_{\mathrm{I}}\right|_{F=\frac{1}{2}}=-\frac{e \sin \theta}{\pi}\left(r_{\max }+r_{0} \ln \frac{r_{\max }}{r_{0}}\right)+O\left(r_{\max }^{-2(v-1)}\right), \quad \frac{3}{5}<v<2
\end{aligned}
$$


and

$$
\begin{aligned}
\left.\Phi_{\mathrm{I}}\right|_{F=v-1} & =-\frac{e}{2 \pi} r_{\max }\left[\frac{\pi}{v} \sum_{p=1}^{\nabla v / 2 \rrbracket} \frac{\sin (3 p \pi / v)}{\sin ^{2}(p \pi / v)}\right. \\
& \left.+\sin (v \pi) \int_{0}^{\infty} \frac{d u}{\cosh ^{2}(u / 2)} \frac{\cosh \left(\frac{3}{2} u\right)}{\cosh (v u)-\cos (v \pi)}\right]+\left\{\begin{array}{l}
O\left(\sqrt{r_{\max }} \ln r_{\max }\right), \quad v=2 \\
O\left(r_{\max }^{-2 v+3}\right), \quad 2<v<7
\end{array}\right\}
\end{aligned}
$$

\section{Conclusions}

Quantum vacuum effects that are due to non-Euclidean geometry of nanocones are studied in the present paper. On the basis of the continuum model for long-wavelength electronic excitations originating in the tight-binding approximation for the nearest-neighbor interactions of the lattice atoms, we consider quantum ground-state effects in monolayers warped into nanocones by a disclination; the nonzero size of the disclination at the apex of a nanocone is taken into account. We show that the $R$-current circulating around the disclination is induced in the ground state; see Equations (26)-(29) and (37). The pseudomagnetic field strength, which is orthogonal to the nanocone surface, is also induced in the ground state; see Equations (30)-(33) and (38). Both the current and the field strength are invariant under time reversal and consist of two parts: one is independent of the disclination size, $r_{0}$, and the other depending on $r_{0}$ is damped at large distances from the apex. In the physically sensible case, that is, at $r_{\max } \gg r_{0}$, the latter part is negligible, and we arrive at the conclusion that quantum ground-state effects are independent of $r_{0}$. Moreover, in this case, the field strength is proportional to the current; see Equation (43), with $\Phi_{\mathrm{I}}$ being the total flux through a nanocone with radial size $r_{\max }$; see Equations (44)-(47).

Our results are relevant for the two-dimensional Dirac materials of conical shape. In particular, for the case of the carbon monolayer (graphene) warped into a nanocone by a disclination, that is, a $\left(6-N_{d}\right)$-gonal $\left(N_{d} \neq 0\right)$ defect inserted in the otherwise perfect two-dimensional hexagonal lattice, the results can be summarized as follows. The dominating contribution to the induced ground-state flux of the pseudomagnetic field through carbon nanocones with $N_{d}= \pm 1, \pm 2, \pm 3$, $4,5,-6$ is

$$
\begin{gathered}
\left.\Phi_{\mathrm{I}}\right|_{\theta \neq-\frac{\pi}{2}}=-\frac{e}{2 \pi} r_{\max } \int_{0}^{\infty} \frac{d u}{\cosh ^{2}(u / 2)} \frac{\sin \left(\frac{1}{5} \pi\right) \cosh \left(\frac{9}{10} u\right)-\sin \left(\frac{7}{5} \pi\right) \cosh \left(\frac{3}{10} u\right)}{\cosh \left(\frac{6}{5} u\right)-\cos \left(\frac{6}{5} \pi\right)}, N_{d}=1 \\
\left.\Phi_{\mathrm{I}}\right|_{\theta=-\frac{\pi}{2}}=\frac{e}{2 \pi} r_{\max } \sin \left(\frac{1}{5} \pi\right) \int_{0}^{\infty} \frac{d u}{\cosh ^{2}(u / 2)} \frac{\cosh \left(\frac{3}{2} u\right)}{\cosh \left(\frac{6}{5} u\right)-\cos \left(\frac{6}{5} \pi\right)}, N_{d}=1 \\
\left.\Phi_{\mathrm{I}}\right|_{\theta \neq \frac{\pi}{2}}=\frac{e}{2 \pi} r_{\max } \int_{0}^{\infty} \frac{d u}{\cosh ^{2}(u / 2)} \frac{\sin \left(\frac{5}{7} \pi\right) \cosh \left(\frac{9}{14} u\right)+\sin \left(\frac{1}{7} \pi\right) \cosh \left(\frac{3}{14} u\right)}{\cosh \left(\frac{6}{7} u\right)-\cos \left(\frac{6}{7} \pi\right)}, N_{d}=-1 \\
\left.\Phi_{\mathrm{I}}\right|_{\theta=\frac{\pi}{2}}=-\frac{e}{2 \pi} r_{\max } \int_{0}^{\infty} \frac{d u}{\cosh ^{2}(u / 2)} \frac{\sin \left(\frac{5}{7} \pi\right) \cosh \left(\frac{15}{14} u\right)-\sin \left(\frac{11}{7} \pi\right) \cosh \left(\frac{3}{14} u\right)}{\cosh \left(\frac{6}{7} u\right)-\cos \left(\frac{6}{7} \pi\right)}, N_{d}=-1 \\
\left.\Phi_{\mathrm{I}}\right|_{\theta \neq-\frac{\pi}{2}}=-\frac{e \sqrt{3}}{4 \pi} r_{\max } \int_{0}^{\infty} \frac{d u}{\cosh (u / 2)} \frac{e \sin \theta}{\cosh \left(\frac{2}{3} u\right)-\cos \left(\frac{2}{3} \pi\right)}, N_{d}=-3
\end{gathered}
$$




$$
\begin{gathered}
\left.\Phi_{\mathrm{I}}\right|_{\theta=-\frac{\pi}{2}}=\frac{e \sqrt{3}}{4 \pi} r_{\max } \int_{0}^{\infty} \frac{d u}{\cosh ^{2}(u / 2)} \frac{\cosh \left(\frac{5}{6} u\right)+\cosh \left(\frac{1}{6} u\right)}{\cosh \left(\frac{2}{3} u\right)-\cos \left(\frac{2}{3} \pi\right)}, N_{d}=-3 \\
\Phi_{\mathrm{I}}=\frac{e}{4} r_{\max }, N_{d}=3 \\
\Phi_{\mathrm{I}}=0, N_{d}=4, \\
\Phi_{\mathrm{I}}=-\frac{e}{4} r_{\max }, N_{d}=5
\end{gathered}
$$

We conclude that the quantum ground-state effects change drastically as $N_{d}$ changes. The effects are absent in the case of the four-heptagonal defect $\left(N_{d}=4\right)$, whereas they appear in the same amount but in opposite sign as a heptagon is removed $\left(N_{d}=3\right)$ or added $\left(N_{d}=5\right)$; see Equations (55)-(57). These cases are independent of the boundary parameter, $\theta$; we note that, namely, these cases correspond to that situation with the zero-size defect when there is no need for self-adjoint extension (the deficiency index is $(0,0)$ ). In all other cases, the results depend on $\theta$. The most distinct dependence is characteristic for the cases of two-pentagonal, and two- and six-heptagonal defects, when the results coincide; see Equation (52); we note that the electric charge is not induced in these cases [11]. In the cases of one-pentagonal, and one- and three-heptagonal defects, the results are almost independent of $\theta$ unless $\theta=-\frac{\pi}{2}$ for $N_{d}=1,-3$ and $\theta=\frac{\pi}{2}$ for $N_{d}=-1$; see Equations (48)-(51), (53) and (54).

Freely suspended samples of crystalline monolayers are not exactly plane surfaces but possess ripples that produce pseudomagnetic fields causing strains and scattering of electronic excitations in a sample [17]. As follows from the present consideration, pseudomagnetic fields can be induced in the locally flat regions out of disclinations, and this may have observable consequences in experimental measurements, likely with the use of scanning tunnel and transmission electron microscopy.

Acknowledgments: Y.A.S. would like to thank the organizers of the 10th Bolyai-Gauss-Lobachevsky Conference on Non-Euclidean Geometry and its Applications for kind hospitality during this interesting and inspiring meeting.

Author Contributions: Y.A.S. formulated tasks and performed calculations; V.M.G. performed calculations.

Conflicts of Interest: The authors declare no conflict of interest.

\section{References}

1. Abrikosov, A.A. On the Magnetic Properties of Superconductors of the Second Group. J. Exp. Theor. Phys. 1957, 5, 1174-1182.

2. Nielsen, H.B.; Olesen, P. Vortex-line models for dual strings. Nucl. Phys. B 1973, 61, 45-61.

3. Vilenkin, A.; Shellard, E.P.S. Cosmic Strings and Other Topological Defects; Cambridge University Press: Cambridge, UK, 2000; p. 517.

4. Hindmarsh, M.B.; Kibble, T.W.B. Cosmic strings. Rep. Prog. Phys. 1995, 58, 477-562.

5. Novoselov, K.S.; Jiang, D.; Schedin, F.; Booth, T.J.; Khotkevich, V.V.; Morozov, S.V.; Geim, A.K. Two-dimensional atomic crystals. Proc. Nat. Acad. Sci. USA 2005, 102, 10451-10453.

6. Geim, A.K.; Novoselov, K.S. The rise of graphene. Nat. Mater. 2007, 6, 183-191.

7. Cahangirov, S.; Topsakal, M.; Akturk, E.; Sahin, H.; Ciraci, S. Two- and One-Dimensinonal Honeycomb Structures of Silicon and Germanium. Phys. Rev. Lett. 2009, 102, 236804.

8. Wehling, T.O.; Black-Schaffer, A.M.; Balatsky, A.V. Dirac materials. Adv. Phys. 2014, 63, 1-76.

9. Battye, R.A.; Garbrecht, B.; Moss, A.; Stoica, H. Constraints on Brane Inflation and Cosmic Strings. J. Cosmol. Astropart. Phys. 2008, 2008, 020.

10. Heiberg-Andersen, H. Carbon nanonones. In Handbook of Theoretical and Computational Nanotechnology; Rieth, M., Schommers, W., Eds.; American Scientific Publishers: Valencia, CA, USA, 2006; Volume 8, pp. 507-517.

11. Sitenko, Y.A.; Vlasii, N.D. Electronic properties of graphene with a topological defect. Nucl. Phys. B 2007, $787,241-259$.

12. Sitenko, Y.A.; Vlasii, N.D. Vacuum polarization effects on graphitic nanocones. J. Phys. Conf. Ser. 2008, 129, 012008. 
13. Sitenko, Y.A.; Vlasii, N.D. Vacuum polarization in graphene with a topological defect. Low Temp. Phys. 2008, 34, 826-833.

14. Neumann, J.V. Mathematische Grundlagen der Quantummechanik; Springer: Berlin, Germany, 1932.

15. Reed, M.; Simon, B. Methods of Modern Mathematical Physics II: Fourier Analysis, Self-Adjointness; Academic Press: Cambridge, MA, USA, 1975; p. 361.

16. Johnson, K. The M.I.T. Bag Model. Acta Phys. Pol. B 1975, 6, 865-919.

17. Vozmediano, M.A.H.; Katsnelson, M.I.; Guinea, F. Gauge fields in grahene. Phys. Rep. 2010, 496, 109-148.

(C) 2018 by the authors. Licensee MDPI, Basel, Switzerland. This article is an open access article distributed under the terms and conditions of the Creative Commons Attribution (CC BY) license (http://creativecommons.org/licenses/by/4.0/). 